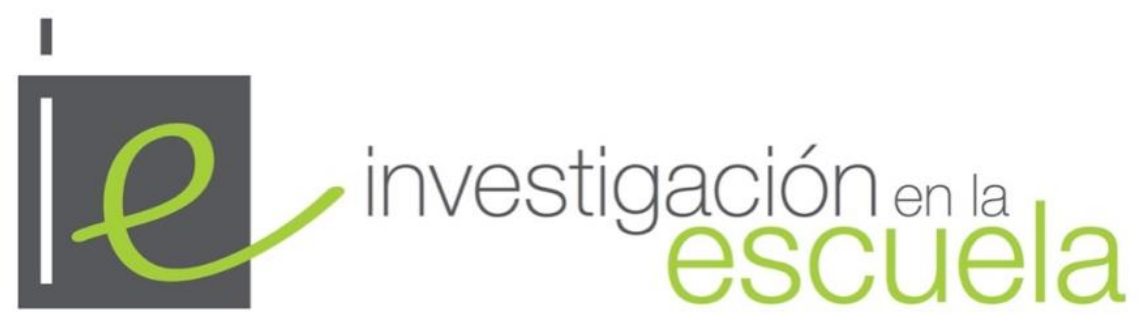

Revista de Investigación e Innovación Educativa nº 102, 2020 | e-ISSN 2443-9991

\title{
Futuros maestros: qué piensan sobre su formación en ciencias y qué hacen en sus prácticas escolares
}

Future teachers: what they think about their science education and what they do in their school practices

iD Dra. Magdalena Valverde Pérez es Profesora Asociada en la Facultad de Educación de la Universidad de Murcia (España)·mvp@um.es·https://orcid.org/0000-0001-6188-6763

iD Dr. Gaspar Sánchez Blanco es Profesor Titular en la Facultad de Educación de la Universidad de Murcia (España)·gsblanco@um.es·https://orcid.org/0000-0002-6186-3366

Cómo citar este artículo

Valverde Pérez, M. y Sánchez Blanco, G. (2020). Futuros maestros: qué piensan sobre su formación en ciencias y qué hacen en sus prácticas escolares. Investigación en la Escuela, 102, 122-139. doi: http://doi.org/10.12795/IE.2020.i102.09

Resumen. En el contexto de la formación inicial de maestros, se estudian los logros del programa en didáctica de las ciencias experimentales, incluyendo la utilidad que tiene para promover cambios docentes durante las prácticas escolares. Concretamente, hemos preguntado a los alumnos por los contenidos y actividades de formación del programa, sus ideas sobre la enseñanza de las ciencias en primaria y sobre lo que hacen cuando enseñan ciencias durante sus prácticas escolares. Para ello, utilizando dos cuestionarios, hemos recogido las opiniones de 131 alumnos de tercer curso del Grado de Educación Primaria al finalizar su formación en ciencias y sus prácticas escolares. Los resultados muestran una valoración muy positiva del programa en didáctica de ciencias, con un claro posicionamiento de los alumnos sobre la enseñanza de las ciencias en primaria coherente con sus presupuestos teóricos, aunque persisten algunas ideas propias del modelo transmisivo en sus planteamientos metodológicos, así como, escasa incidencia en las actuaciones docentes que llevan a cabo durante las prácticas escolares. En ese contexto, el modelo de enseñanza tradicional percibido por los futuros maestros en las aulas y su bajo nivel de autoeficacia para realizar prácticas docentes innovadoras, parecen dificultar la conexión entre los conocimientos académicos y la práctica profesional.

Abstract. Within the context of the initial training of future teachers, the achievements of the program in Experimental Science teaching are assessed, including its usefulness to promote teaching changes during school practices. Specifically, students were asked about the contents and training activities of the program, their ideas about teaching science in Primary school and about what they do when they teach science during their school practices. For this purpose, the opinions of 131 future teachers who were in the 3rd. year of the Degree of Primary Education were collected by means of two questionnaires administrated at the end of their science training and school practices. Results show a very positive assessment of the Science teaching program. Future teachers maintain a clear position on Science education in Primary school consistent with the theoretical assumptions of Science program. On the other hand, the incidence of the teaching program in their teaching actions performed during school practices is scarce. In this context, the traditional teaching model perceived by future teachers in the classrooms, and their low level of self-efficacy to develop innovative teaching practices, seem to make it difficult to connect academic knowledge to professional practice.

\section{Palabras clave $\cdot$ Keywords}

Formación inicial de maestros, didáctica de ciencias experimentales, programa de formación, prácticas escolares, enseñanza de ciencias, educación primaria, concepciones docentes, práctica docente.

Initial teacher training, science education, training courses, school practices, experimental science teaching, primary school; ideas teaching, actions teaching. 


\section{Introducción}

La incorporación de los estudios de formación de maestros en el Espacio Europeo de Educación Superior ha supuesto cambios en los planes de estudios relacionados con su extensión, inclusión de competencias, desaparición de especialidades, ampliación de las prácticas en los centros educativos,... (Bozu y Aránega, 2017) que ha conllevado una reestructuración del programa de formación en Didáctica de Ciencias Experimentales (DCCEE).

Numerosas investigaciones en DCCEE señalan que los estudiantes que acceden al Grado de Educación Primaria presentan serias deficiencias formativas, destacando un escaso conocimiento científico y unas concepciones transmisivas sobre la enseñanza y aprendizaje de las ciencias, influidas por sus experiencias personales como alumnos; así como dificultad de cambiar dichas concepciones (Mellado, 1998a; Cañal, 2000; Pujol, 2008; Porlán et al., 2010; Porlán et al., 2011; García-Carmona et al., 2014; García-Carmona y Cruz, 2016). Las posibles causas de esta dificultad tienen que ver con barreras "externas" a la propia actividad formativa (excesivo número de alumnos por aula o la escasa integración con la práctica docente) y obstáculos "internos" relacionados con aspectos epistemológicos (visiones de los estudiantes sobre la ciencia, la investigación, la enseñanza y el aprendizaje), así como, con niveles de autoeficacia percibida (conocimiento de las estrategias de enseñanza y las habilidades para gestionar el desarrollo de la clase, implicar a los estudiantes o establecer buenas relaciones con ellos, como la definen Blonder et al., (2014)), que dificultan seriamente las soluciones que desde la DCCEE se dan para implementar en las aulas de primaria nuevos enfoques metodológicos que superen el aprendizaje memorístico de datos y hechos (Gil Flores, 2016; Goddard et al., 2004; Mellado, 2001; Smith y Southerland, 2007; Porlán et al., 2010).

Generalmente, en la DCCEE, se acepta que la formación de un profesor debe partir de sus conocimientos docentes iniciales y que se han de proporcionar oportunidades para facilitar su evolución (Mellado y González, 2000; Porlán et al., 2010; Bonil y Márquez, 2011). En este sentido, el programa de formación tendrá que seleccionar y estructurar numerosos conocimientos teórico-prácticos relativos a finalidades educativas, contenidos de enseñanza, dificultades de aprendizaje, métodos de enseñanza y de evaluación, así como hacer posible el diseño y desarrollo de situaciones didácticas (actividades y/o lecciones) que promuevan mejoras en la práctica docente. Situaciones didácticas que deberían poder implementarse durante sus prácticas escolares (PE) para propiciar la reflexión sobre el proceso de enseñanza/aprendizaje de las ciencias y, mediante esta implicación personal activa completa, comenzar a adquirir rutinas y estrategias que hagan posible cambios en el conocimiento didáctico del contenido (CDC) que utilizan los profesores en sus aulas (Mellado, 1999; Sánchez y Valcárcel, 2000).

En el contexto de las PE, los trabajos de investigación se sitúan en el estudio de la mejora del diseño y calidad de las prácticas (Ruiz-Bernardo et al., 2018; Tuli y File, 2009), efectos que determinados contenidos o estrategias adoptados en la formación tienen sobre la autoeficacia docente percibida (Gunning y Mensah, 2011; Richardson y Lian, 2008; Petersen y Treagust, 2014; Velthuis et al., 2013), y en el contexto de la enseñanza de las ciencias, en lo que piensan los futuros maestros sobre las clases de ciencias y lo que hacen cuando enseñan (Cañal et al., 2013; De Pro y Nortes, 2016; Cortés et al., 2012; Escobar y Vílchez, 2008; King et al., 2001; Martínez-Losada et al., 2017; Parker y Spink, 1997; Vílchez y Escobar, 2014). Sin embargo, la mayoría se centran más en las percepciones y valoraciones de lo que se hace y no tanto en las actuaciones que se llevan a cabo.

Por tanto, tras varios cursos académicos con el actual programa de formación en DCCEE y considerar insuficiente los trabajos que profundizan en la posible incidencia que tiene en las PE, nos planteamos tres cuestiones: ¿Cómo valoran los futuros maestros el programa de formación? ¿Qué piensan sobre la enseñanza de las ciencias en primaria? ¿Qué hacen en las PE cuando enseñan ciencias en primaria? Estos interrogantes nos permiten establecer como objetivos específicos:

- Valorar la utilidad que los alumnos le asignan a los conocimientos y actividades formativas del programa de formación de DCCEE para su futura labor docente.

- Conocer las concepciones de los estudiantes sobre la enseñanza de las ciencias en primaria al finalizar el programa de formación en DCCEE.

- Conocer qué utilizan del programa en DCCEE y qué aplican de sus concepciones sobre enseñar ciencias en sus actuaciones docentes durante las PE. 


\section{Contexto de la formación: Didáctica de Ciencias Experimentales y Prácticas Escolares}

El programa de formación en DCCEE del Grado de Educación Primaria de la Universidad de Murcia (GEPUM) tiene como finalidad dotar a los futuros maestros de un CDC sobre las ciencias, en general, y sobre los bloque temáticos habituales del currículo de educación primaria, en particular, que haga posible cambiar las concepciones docentes iniciales de los alumnos y el desarrollo de conocimientos, destrezas y habilidades docentes relacionadas con el análisis y diseño de situaciones didácticas que, en primera instancia, sea útil en las PE y, en último término, sea el principal referente para innovar y mejorar la enseñanza de las ciencias en la escuela (Cañal, 2000; Mellado y González, 2000; Pujol, 2008; Porlán et al., 2010). En él se incluyen tres asignaturas, de 6 créditos, impartidas en dos cursos consecutivos del plan de estudios $\left(2^{\circ}\right.$ y $\left.3^{\circ}\right)$. La primera asignatura, Didáctica de las Ciencias Experimentales en Educación Primaria (DCCEEP), de carácter introductorio y básico, aborda diferentes temáticas que deben contemplarse durante la planificación de la enseñanza de las ciencias: finalidades educativas, contenidos de enseñanza, problemas de aprendizaje, estrategias didácticas y de evaluación. Las otras dos asignaturas, Enseñanza y Aprendizaje del Medio Natural I (EAMN-I) y Enseñanza y Aprendizaje del Medio Natural II (EAMN-II), abordan el tratamiento escolar de los bloques de contenido del currículo de educación primaria. En relación con cada tópico, se trabajan y actualizan los contenidos escolares, se plantean y discuten dificultades de su aprendizaje, y se analizan y diseñan propuestas de enseñanza (actividades y/o lecciones). Las tres asignaturas comparten el tipo de actividades formativas (tabla 1).

\section{Tabla 1}

Actividades formativas compartidas por las asignaturas del programa en DCCEE

\begin{tabular}{|c|c|c|}
\hline $\begin{array}{l}\text { Actividad } \\
\text { Formativa }\end{array}$ & Descripción & Metodología \\
\hline $\begin{array}{l}\text { Clases } \\
\text { teóricas }\end{array}$ & $\begin{array}{l}\text { Junto a la exposición oral de los contenidos básicos de cada tema } \\
\text { del programa se plantean cuestiones o problemas, se aclaran } \\
\text { dudas, se realizan ejemplificaciones, se establecen relaciones con } \\
\text { las actividades prácticas o trabajos que se planteen, se orienta la } \\
\text { búsqueda de información,.... } \\
\text { Se desarrollan paralelamente a las exposiciones del profesor. }\end{array}$ & Clases expositivas \\
\hline $\begin{array}{l}\text { Actividades } \\
\text { prácticas de } \\
\text { aula }\end{array}$ & $\begin{array}{l}\text { Realización por parte del alumnado de actividades individuales o } \\
\text { en grupo, para explicitar las ideas iniciales, generar nueva } \\
\text { información, utilizar nuevos conocimientos y poner en común } \\
\text { conclusiones }\end{array}$ & Prácticas de aula \\
\hline Seminarios & $\begin{array}{l}\text { Sesiones de trabajo colaborativo entre alumnos, realizadas en } \\
\text { pequeños grupos y supervisadas por el profesor. Mediante el } \\
\text { análisis de cualquier material curricular, o mediante el diseño de } \\
\text { nuevas propuestas de enseñanza, se pretende dar respuesta al } \\
\text { qué, cómo y para qué se enseña, y propiciar una reflexión sobre } \\
\text { la problemática del proceso de enseñanza y aprendizaje y los } \\
\text { marcos teóricos que se proponen desde la DCCEE como } \\
\text { mejores soluciones a dichos problemas. Requieren la } \\
\text { presentación de un informe escrito (individual y/o grupal) para } \\
\text { su evaluación. }\end{array}$ & $\begin{array}{l}\text { Actividades prácticas } \\
\text { en aula convencional }\end{array}$ \\
\hline $\begin{array}{l}\text { Prácticas de } \\
\text { laboratorio }\end{array}$ & $\begin{array}{l}\text { Realización de actividades experimentales por parte de los } \\
\text { alumnos, dirigidas y supervisadas por el profesor, vinculadas a un } \\
\text { saber hacer propio del trabajo científico y realizadas en pequeños } \\
\text { grupos. Requieren la presentación de un informe escrito } \\
\text { (individual y/o grupal) para su evaluación. }\end{array}$ & $\begin{array}{l}\text { Actividades prácticas } \\
\text { en aula especial }\end{array}$ \\
\hline Examen & $\begin{array}{l}\text { Resolución de preguntas teórico-prácticas para evaluar el } \\
\text { dominio de conocimientos teóricos y prácticos adquiridos. }\end{array}$ & Prueba escrita \\
\hline
\end{tabular}

El GEPUM incluye tres periodos de PE, con un enfoque de carácter reflexivo, determinado tanto por el análisis de las acciones docentes observadas como de las realizadas, utilizando contenidos y conocimientos adquiridos de las materias cursadas en el Grado. El segundo periodo de prácticas (PE-II), con una duración de ocho semanas, se sitúa tras completar la formación obligatoria en ciencias y es el que 
forma parte de nuestra investigación. En él intervienen dos tutores, uno de la Facultad de Educación con docencia en áreas de didáctica de materias generalistas del currículo de educación primaria (Didáctica de las Ciencias Experimentales, Didáctica de las Ciencias Sociales, Didáctica de las Matemáticas, Didáctica de la Lengua y la Literatura y Didáctica de la Expresión Plástica), de ahí que el profesorado del área de Didáctica de las Ciencias Experimentales solo pueda dirigir y supervisar las actividades de un número reducido de estudiantes mediante la realización de 4 seminarios de trabajo, siendo esta una de las principales limitaciones del programa de formación; otro, un maestro del centro escolar que organiza el desarrollo de las actividades que deben realizar los estudiantes y ayuda en el desempeño de la función docente. Ambos tutores participan en la evaluación de los alumnos. Durante las prácticas, los estudiantes deben diseñar, poner en práctica y evaluar tres actividades puntuales de diferentes áreas del currículo de Educación Primaria para una sesión de clase, concretando para un tópico, contenidos de enseñanza, objetivos, método de enseñanza, evaluación y competencias a las que contribuye; así como, una unidad de programación de una de las áreas no impartidas por especialistas, y de la asignatura del tutor de Facultad (si este así lo aconseja), prevista en la programación de aula para el periodo de prácticas. La selección de actividades debe hacerse de acuerdo con ambos tutores.

\section{Metodología}

\subsection{Contexto de la investigación}

La fuente de datos de esta investigación procede de dos cuestionarios cumplimentados por dos grupos de estudiantes de 3er curso del GEPUM tras finalizar el programa de formación en DCCEE y completar las PE, en los cursos 2016/17 (cuestionario CF1) y 2017/18 (cuestionario CF2).

Participa un total de 131 estudiantes, 72 de ellos cumplimentan el cuestionario CF1 con el fin de conocer sus actuaciones docentes en ciencias durante las PE, y los otros 59, responden al cuestionario CF2 para relacionar sus actuaciones docentes con la formación recibida en DCCEE y sus concepciones sobre enseñar ciencias. Ambos cuestionarios se enviaron mediante el aula virtual a una población similar de sujetos quienes podían responder opcionalmente. Las características de los dos grupos de participantes se recogen en la tabla 2.

Tabla 2

Características de los grupos de estudiantes que responden a los cuestionarios CF1 y CF2. Valores expresados como frecuencia (\%)

\begin{tabular}{lcccccc}
\hline & \multirow{2}{*}{ Edad } & \multicolumn{2}{c}{ Género } & \multicolumn{3}{c}{$\begin{array}{c}\text { Alumnos que superan las asignaturas } \\
\text { programa de formación en DCCEE }\end{array}$} \\
\hline Grupo & $20-22$ años & Hombre & Mujer & DCCEEP & EAMN-I & EAMN-II \\
CF1 & $61(84,7)$ & $16(22,2)$ & $56(77,8)$ & $71(98,6)$ & $63(92,6)$ & $45(67,2)$ \\
CF2 & $45(76,3)$ & $14(23,7)$ & $45(76,3)$ & $59(100,0)$ & $54(91,5)$ & $39(66,1)$ \\
\hline
\end{tabular}

Las PE las han realizado en diversos centros educativos de la Región de Murcia. El curso asignado para realizar las PE y el idioma en el que se imparten las ciencias (CC) se presenta en la tabla 3, destacando la heterogeneidad en el curso donde realizan las PE, así como los altos porcentajes de alumnos en aulas donde las ciencias (CC) se imparten en inglés.

Tabla 3

Curso asignado e idioma en que se imparten las ciencias. V alores expresados como frecuencia (\%)

\begin{tabular}{lcccccccc}
\hline \multicolumn{7}{c}{ Curso asignado } & \multicolumn{3}{c}{ CC se imparten } \\
\hline \multirow{2}{*}{ Grupo } & $1^{\circ}$ & $2^{\circ}$ & $3^{\circ}$ & $4^{\circ}$ & $5^{\circ}$ & $6^{\circ}$ & Españo & Inglés \\
& 14 & 12 & 13 & 14 & 8 & 11 & 37 & $35(48,6)$ \\
CF1 & $(19,4)$ & $(16,7)$ & $(18,1)$ & $(19,4)$ & $(11,1)$ & $(15,3)$ & $(51,4)$ & \\
\multirow{2}{*}{ CF2 } & 14 & 10 & 6 & 6 & 12 & 11 & 41 & $18(30,5)$ \\
& $(23,7)$ & $(16,9)$ & $(10,2)$ & $(10,2)$ & $(20,3)$ & $(18,6)$ & $(69,5)$ & \\
\hline
\end{tabular}




\subsection{Instrumento de investigación}

Como se ha señalado, para la recogida de información se diseñaron dos cuestionarios "ad-hoc". El cuestionario CF1 incluye 4 ítems con los que se analizará: el tipo de actuación docente, contenido trabajado, actuación docente donde aplica la formación recibida en DCCEE y para qué utilizan el conocimiento académico en DCCEE es sus actuaciones. Con el cuestionario CF2 analizaremos: cómo valoran los contenidos teóricos y prácticos y actividades y tareas formativas del programa de formación, cuáles son sus concepciones sobre enseñar ciencias y qué hacen cuando enseñan ciencias en primaria. La tabla 4 recoge los ítems utilizados en la respuesta dada a los problemas de la investigación.

Tabla 4

Intencionalidad y dimensiones

\begin{tabular}{clcc}
\hline Problema & \multicolumn{1}{c}{ Intencionalidad } & Dimensión & Ítem-Cuestionario \\
\hline $\begin{array}{c}\text { PP1: } \\
\text { Programa } \\
\text { formación en } \\
\text { DCCEE }\end{array}$ & $\begin{array}{l}\text { Conocer la utilidad del } \\
\text { programa para su futura labor } \\
\text { docente }\end{array}$ & $\begin{array}{c}\text { Contenidos teóricos y } \\
\text { prácticos } \\
\text { Actividades y tareas } \\
\text { formativas }\end{array}$ & 1-CF2 \\
PP2: & Conocer las concepciones de & Para qué enseñar & 2, 3, 4-CF2 \\
Enseñanza & Qué enseñar & 5-CF2 \\
Ciencias & los alumnos & Cómo enseñar & 6-CF2 \\
& $\begin{array}{l}\text { Conocer sus actuaciones } \\
\text { docentes en ciencias y } \\
\text { relacionar el conocimiento } \\
\text { académico y concepciones } \\
\text { PP3: el conocimiento }\end{array}$ & Qué hacen & 7-CF2 \\
Prácticas & & 8, 9-CF2 \\
Escolares & 1,2-CF1 & \\
& PE & Qué utilizan & 10, 11-CF2 \\
& & & 3, 4-CF1 \\
\hline
\end{tabular}

Los cuestionarios combinan preguntas cerradas de respuesta múltiple, no excluyentes entre sí para algunos ítems (1, 2, 3, 4-CF1 y 8, 9, 10, 11-CF2) o de valoración en una escala Likert de 5 puntos (1, 2, 3, 4, 5, 6, 7-CF2) donde los estudiantes debían mostrar el grado de acuerdo, siendo el 1 el valor mínimo o de completo desacuerdo, 2 desacuerdo, 3 valor que no define desacuerdo o acuerdo, 4 acuerdo y 5 el valor máximo o de completo acuerdo.

\subsection{Análisis de datos}

En el análisis estadístico de los datos relacionados con los problemas PP1 y PP2, las comparaciones entre las afirmaciones (A, B, ..,H) incluidas en cada ítem (1-CF2 hasta 7-CF2), se realizaron utilizando el Modelo Lineal General (MLG) univariante seguido de pruebas de Bonferroni para comparaciones múltiples corregidas cuando el MLG mostró diferencias significativas, estableciéndose un valor de significancia más estricto $(\mathrm{P}<.01)$ y así evitar errores de tipo I. Los cálculos estadísticos se realizaron utilizando el paquete estadístico SPSS.

\section{Resultados y discusión}

Situados en un marco declarativo mediante respuestas a cuestionarios, se exponen los resultados más relevantes del estudio. 


\section{1. ¿Cómo valoran los alumnos el programa de formación?}

Al finalizar el programa de formación en ciencias, los alumnos expresan un grado de acuerdo alto en la utilidad del programa para aprender a enseñar ciencias, con una valoración media de 4,07. Las comparaciones post-hoc de Bonferroni reportan que, el tipo de actividades y tareas formativas del programa (ítem 2-CF2) son las que menor utilidad significativamente le otorgan para enseñar ciencias ( $\mathrm{p}<$ .01) con un valor de 3,88, y los tres ítems mejor puntuados son 1-CF2, 3-CF2 y 4-CF2 (con una valoración superior a 4) (tabla 5).

Tabla 5

Valoración global del programa de formación en ciencias

\begin{tabular}{lccccc}
\hline $\begin{array}{l}\text { Ítem- } \\
\text { Cuestionario }\end{array}$ & $\begin{array}{c}\text { 1-CF2. } \\
\text { Contenidos } \\
\text { teóricos y } \\
\text { prácticos }\end{array}$ & $\begin{array}{c}\text { 2-CF2. } \\
\text { Actividades } \\
\text { y tareas } \\
\text { formativas }\end{array}$ & $\begin{array}{c}\text { 3-CF2. } \\
\text { Análisis y } \\
\text { diseño } \\
\text { propuestas } \\
\text { enseñanza }\end{array}$ & $\begin{array}{c}\text { 4-CF2. } \\
\text { Conocimientos } \\
\text { científicos y } \\
\text { didácticos }\end{array}$ & $\begin{array}{c}\text { Programa } \\
\text { Formación }\end{array}$ \\
\hline Media & $4,06 a b(0,88)$ & $3,88 \mathrm{~b}$ & $4,10 \mathrm{a}$ & $4,20 \mathrm{a}$ & 4,07 \\
(SD) & $(1,09)$ & $(0,98)$ & $(0,85)$ & $(0,96)$ \\
\hline
\end{tabular}

\section{Contenidos teóricos y prácticos}

Entre el 61 y 86,5 \% de los alumnos reconocen la utilidad de todos los contenidos teórico-prácticos desarrollados, con valoraciones $\geq 4$, lo que expresa un grado de acuerdo importante para cada afirmación. La comparación por afirmaciones muestra que los contenidos relacionados con los conocimientos científicos y las estrategias de evaluación son los que consideran significativamente de menor utilidad, para enseñar ciencias (figura 1). La menor valoración de la revisión de los conocimientos científicos puede ser explicada atendiendo a que en los alumnos prevalece la idea de que los contenidos escolares son fáciles y no requieren especial preparación, y/o son capaces de autoformarse, resultado de la "concepción del conocimiento escolar como proceso simplificado del conocimiento científico" (Cañal et al., 2008). Por otro lado, el menor interés por la evaluación, como señalan Martínez-Losada et al. (2017), puede deberse a que consideran que viene definido por el currículo y, por tanto, no tienen que tomar decisiones al respecto.

\section{Figura 1}

Valoración de la utilidad de los contenidos teórico-prácticos. * Medias con diferentes letras en superíndice (a,b) difieren significativamente $(P<0,01)$

Ítem 1-CF2: Las tres asignaturas han desarrollado contenidos teóricos y prácticos, ¿cuáles consideras que son más necesarios y útiles para aprender a enseñar ciencias en Primaria?

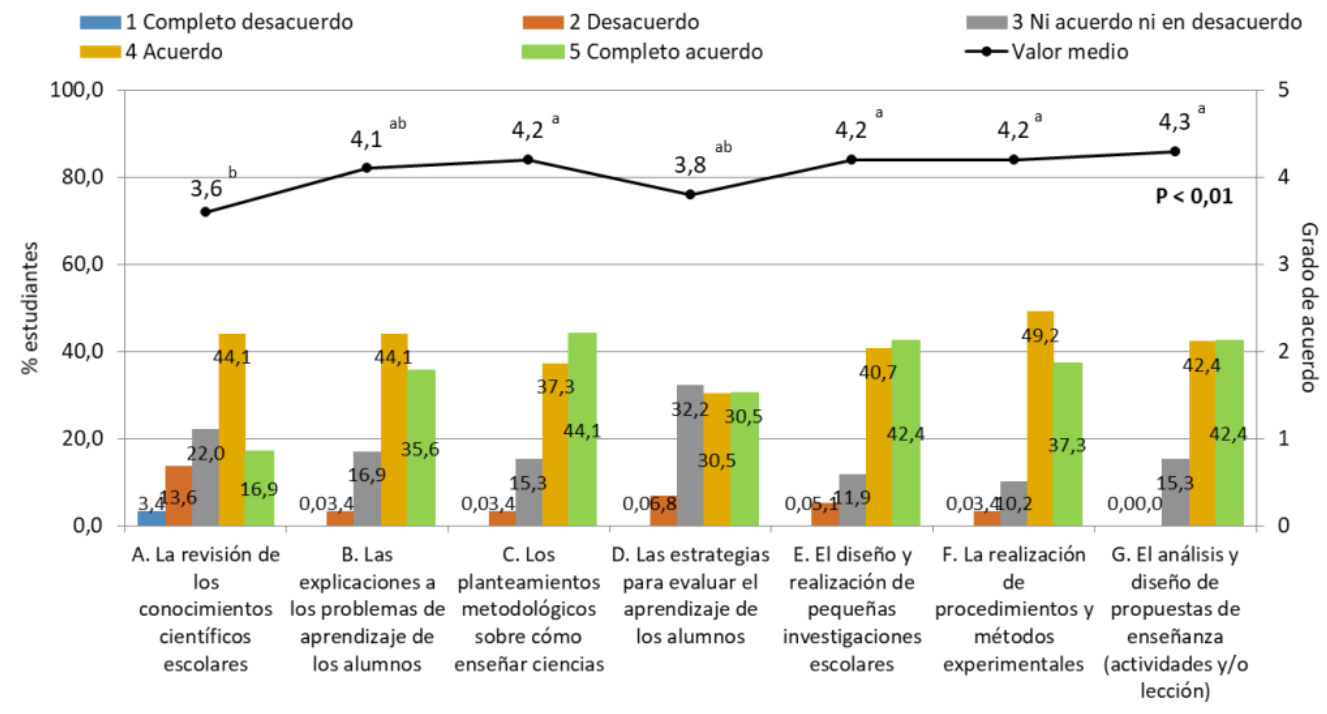


Este planteamiento es coherente con la finalidad del programa de formación, adquirir un conocimiento didáctico suficiente para planificar la enseñanza de las ciencias en primaria, ya que contemplan aspectos teóricos y prácticos que deben orientar su actuación, aunque siguen sin dar la importancia y el interés deseable al conocimiento científico escolar y al proceso de evaluación.

\section{Actividades y tareas formativas}

Del conjunto de actividades formativas, las de mayor éxito son las que desarrollan contenidos prácticos con un respaldo superior al $80 \%$ y una valoración $\geq 4,2$. Por el contrario, el MLG univariante con corrección de Bonferrori indica diferencias significativas para tres de las siete actividades y tareas formativas, reportando las comparaciones post-hoc que las clases teóricas son las peor valoradas para aprender a enseñar ciencias. Las tareas de trabajo autónomo (elaboración informes y examen final) son valoradas positivamente en menor medida (figura 2).

\section{Figura 2}

Valoración de la utilidad de las actividades y tareas formativas. ${ }^{*}$ Medias con diferentes letras en superíndice (a,b,c) difie ren significativamente $(P<0,01)$

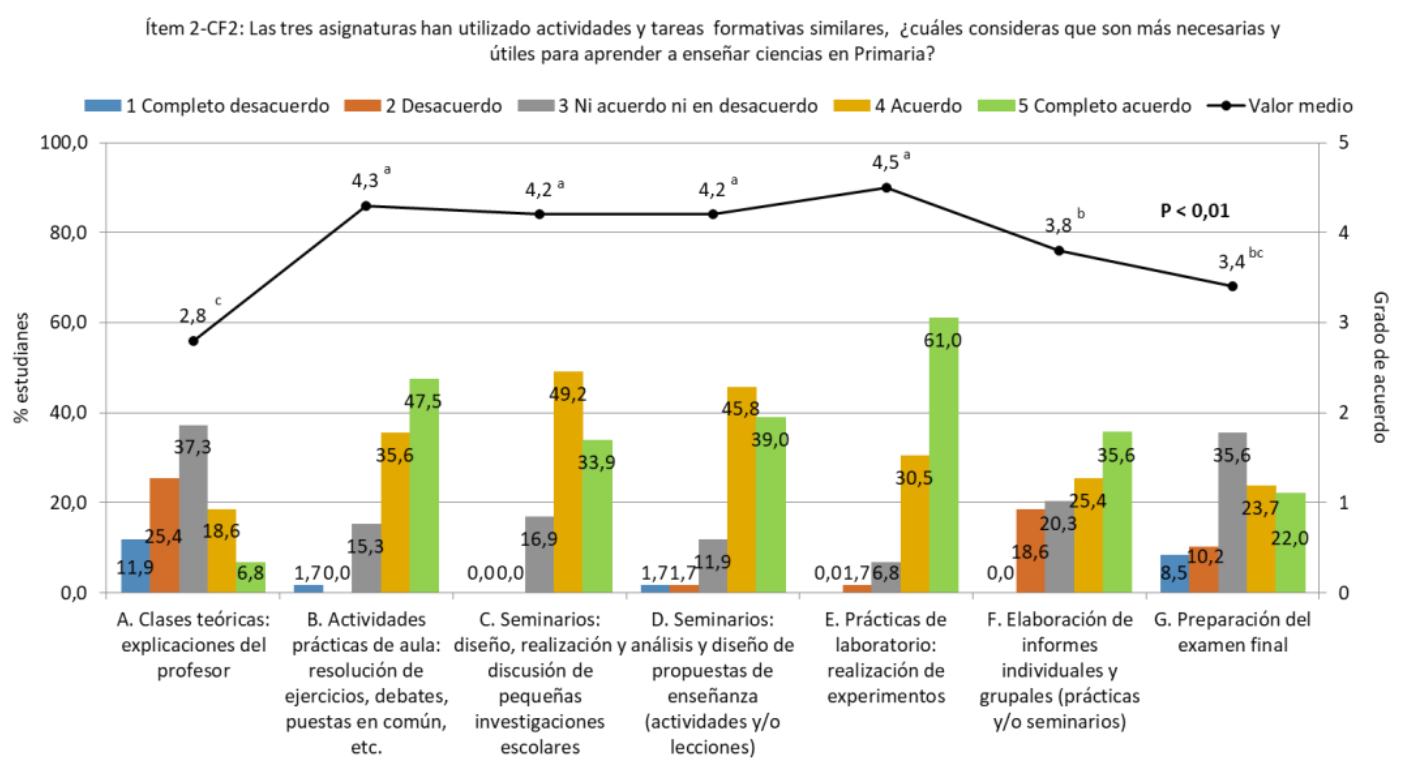

Creemos que los resultados responden, por un lado, a que los alumnos reconocen la necesidad de adquirir conocimientos y destrezas necesarias para su futura labor docente y, por otro, a cómo se sitúan ante las diversas actividades formativas en función de las exigencias o naturaleza más lúdica de las mismas. Así, el desacuerdo e indecisión mostrado por la necesidad y utilidad de las clases teóricas y el examen final podría deberse a exigencias académicas de la formación, al no querer examinarse de contenidos que les son difíciles y que en muchos casos les supone un reto (Cortés et al., 2012). También, como antes señalamos, porque al creer que cuentan con los conocimientos científicos suficientes no admiten reconocer el valor de estas actividades formativas muy dirigidas a su revisión y actualización.

Por el contrario, en el contexto de los seminarios dedicados al análisis y diseño de propuestas de enseñanza, los alumnos reconocen de modo similar, entre el $72-81 \%$, la utilidad de todos los conocimientos necesarios para enseñar ciencias que contempla el programa de formación, incluido tener conocimientos científicos sobre el tema, con valoraciones $\geq 4$ que no difieren significativamente atendiendo al MLG univariante (figura 3). Los resultados dan muestra de la pertinencia de estas actividades formativas que sitúan a los alumnos más directamente en el contexto del aula mediante diferentes tareas propias de la planificación de la enseñanza. 


\section{Figura 3}

Valoración de la utilidad del análisis y diseño de propuestas de enseñanza

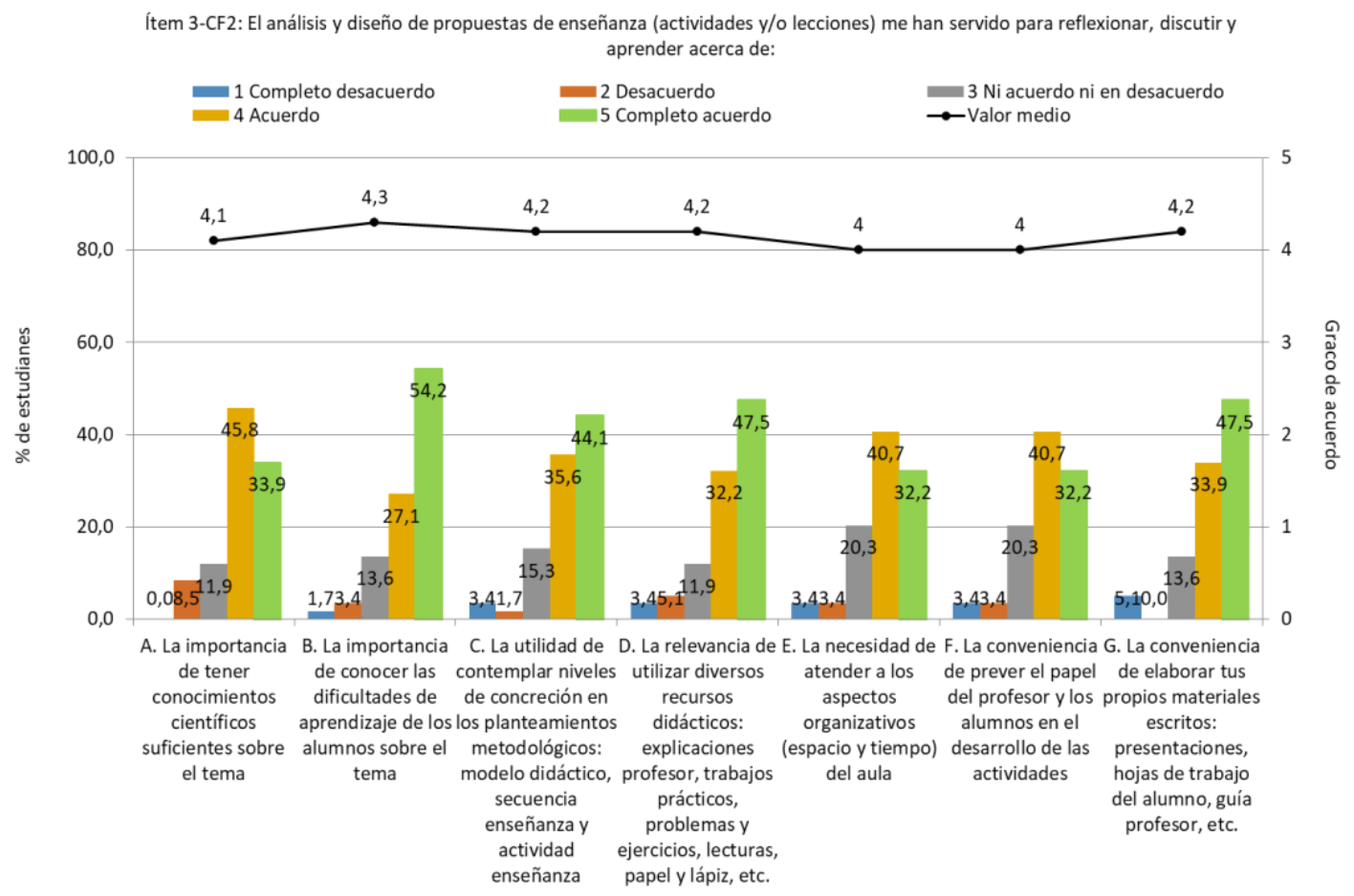

De hecho, cuando se les pregunta directamente por la utilidad del programa en relación con actuaciones docentes que previsiblemente llevarán a cabo cuando enseñen ciencias, los resultados muestran que más del $80 \%$ de los estudiantes incluyen la mayoría de los conocimientos y aprendizajes científicos y didácticos recibidos, con valoraciones $\geq 4$ en todos los casos que no difieren significativamente (figura 4).

\section{Figura 4}

Valoración de la utilidad de los conocimientos científicos y didácticos del programa de formación para ser maestro

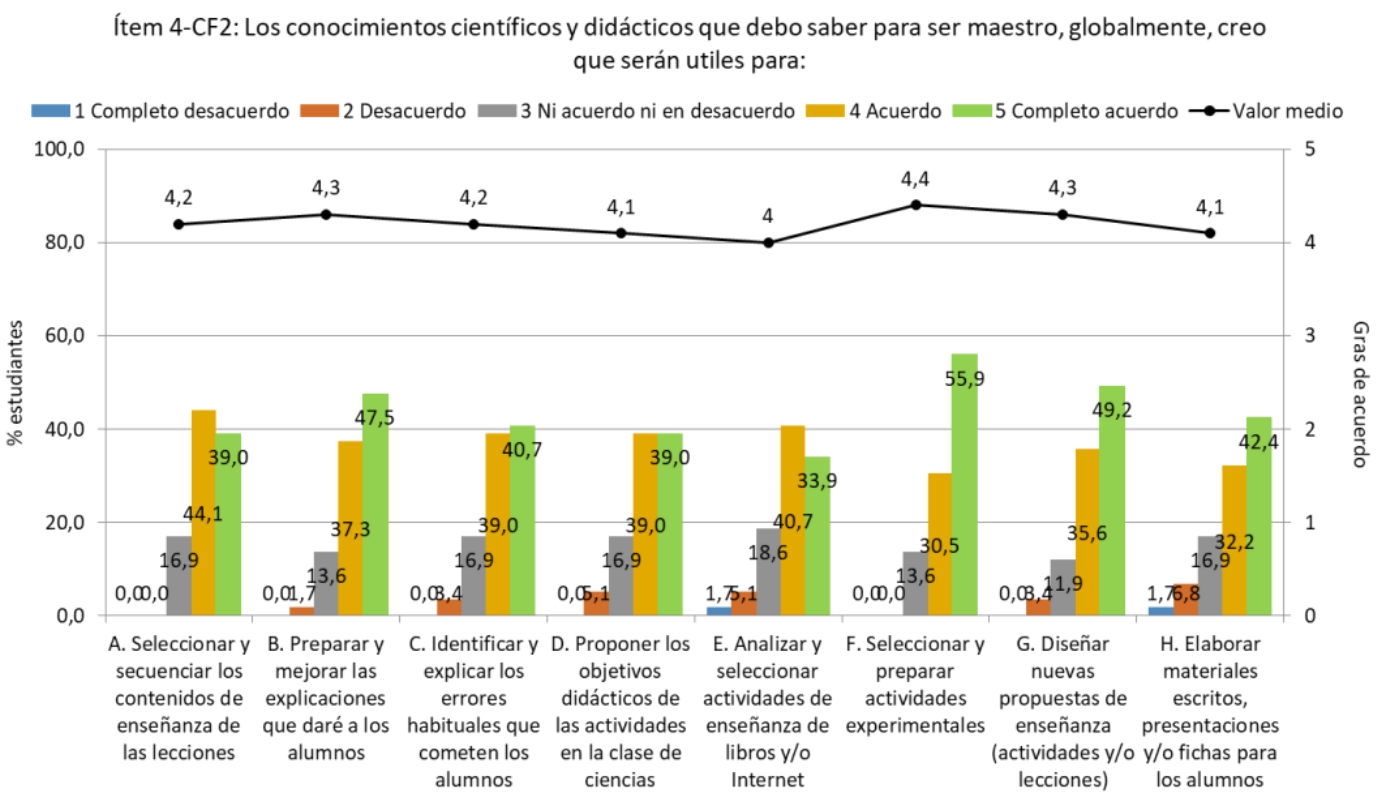

Por tanto, cabría pensar que el desarrollo del programa de formación, en general, y el análisis y diseño de propuestas de enseñanza, en particular, favorece la integración y transformación del conocimiento recibido en $\mathrm{CDC}$ que puede ser útil para iniciar su práctica docente en las $\mathrm{PE}$. 


\section{2. ¿Qué piensan los alumnos sobre la enseñanza de las ciencias en primaria?}

\section{Para qué enseñar ciencias en primaria}

Para más del $80 \%$ de los alumnos las finalidades de la enseñanza de las ciencias en primaria se corresponden con las propuestas en el currículo oficial de educación primaria (Real Decreto 126/2014, de 28 de febrero, por el que se establece el currículo básico de la Educación Primaria), quizás por ser referente durante su formación, y con las recomendaciones realizadas desde la Didáctica de las Ciencias Experimentales (Pedrinaci et al., 2012), al reconocer que los estudios de educación primaria deben favorecer el desarrollo de la competencia científica, mediante la adquisición de capacidades y aprendizajes básicos que contribuyen a las tres dimensiones identificadas por Cañal (2012), conceptual, metodológica y actitudinal (figura 5).

El MLG univariante con corrección de Bonferroni señala que no hay diferencias significativas entre las distintas afirmaciones, pero cabe destacar, que para el $94,9 \%$ y $91,5 \%$ de los futuros maestros (valoraciones $\geq 4$ ) la finalidad de enseñar ciencias en primaria está relacionada con el desarrollo de actitudes hacia el medio ambiente y la adquisición de estrategias de investigación, respectivamente.

\section{Figura 5}

Finalidades de la enseñanza de las ciencias en Primaria

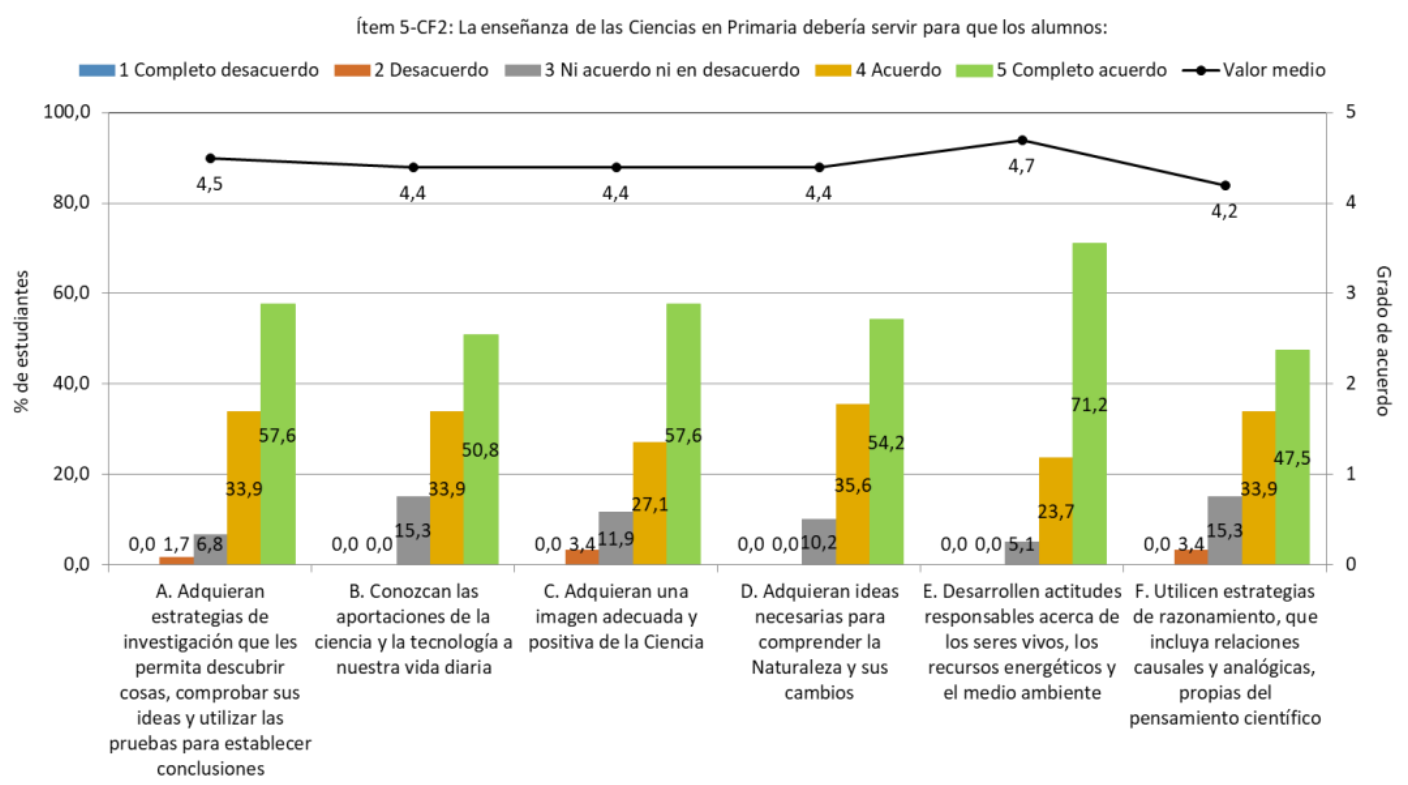

\section{Qué aspectos enseñar de ciencias en primaria}

Al comparar entre las diferentes afirmaciones, el MLG univariante indica diferencias significativas, de forma que, los alumnos comparten una enseñanza de las ciencias donde los conocimientos escolares sean menos dependiente de lo conceptual, inclinándose por conocimientos de ciencia más útiles para una educación integral del alumnado, destacando contenidos relacionados con las actitudes ante las implicaciones sociales de la ciencia y las propias del trabajo científico, así como, con las estrategias y métodos de la investigación científica (figura 6). 


\section{Figura 6}

Contenidos de enseñanza de las ciencias en Primaria. * Medias con diferentes letras en superíndice (a,b,c) difieren significativamente $(P<0,01)$

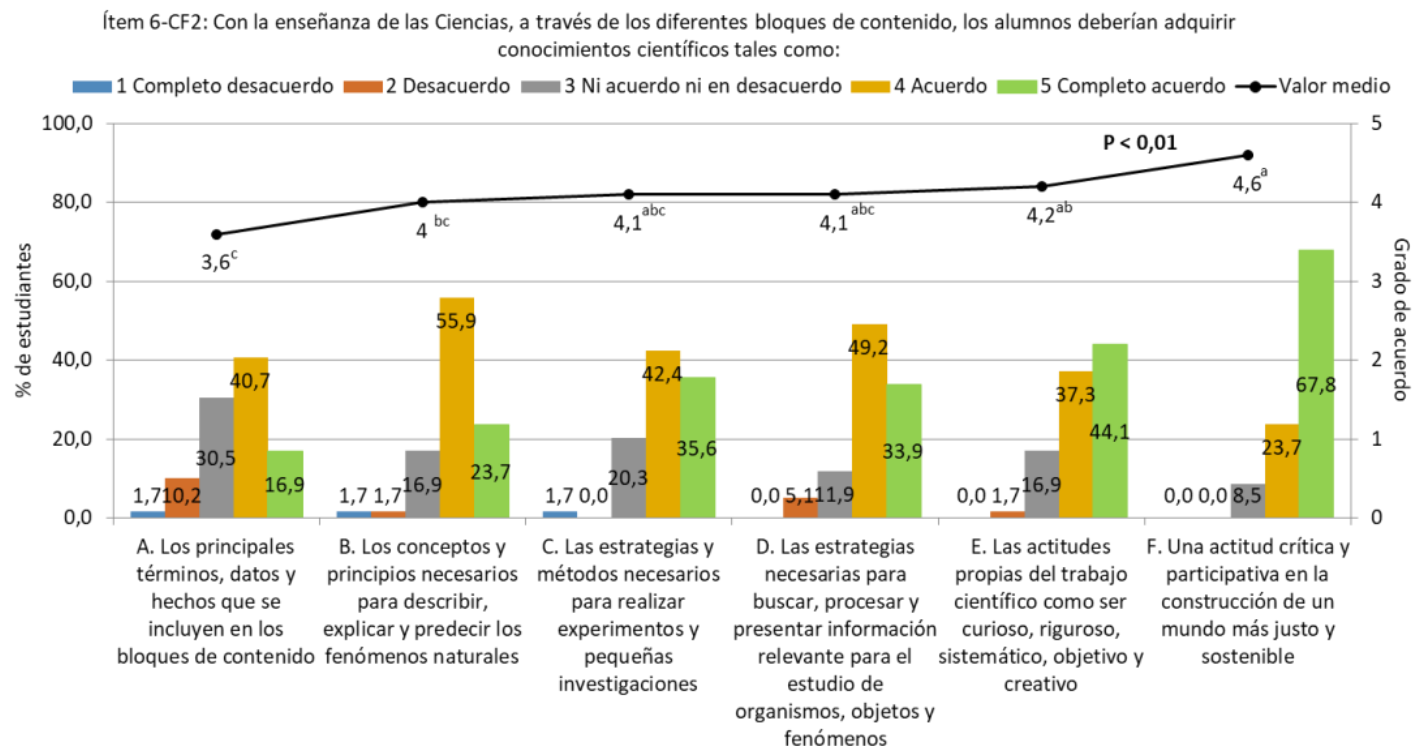

\section{Cómo enseñar ciencias en primaria}

Los futuros maestros rechazan elementos propios de un enfoque tradicional extremo (explicar realizar ejercicios), que difieren significativamente de la opción de enfoques de enseñanza prácticos y centrados en el alumno (más del $76 \%$ con valoraciones $\geq 4$ ). La situación es similar a la descrita por García-Carmona et al. (2014) donde los maestros en formación rechazan enfoques metodológicos tradicionales, que vinculan a aprendizajes disciplinares memorísticos, y se inclinan por enfoques que favorezca el aprendizaje significativo, integrado y funcional (Pedrinaci et al., 2012; Cañal, 2012; GarcíaCarmona y Cruz-Guzmán, 2016). Cabe destacar que un porcentaje importante $(79,7 \%)$ incluye planteamientos activos de corte transmisivo (explicar el tema y utilizar experimentos para comprobar la teoría) (figura 7).

\section{Figura 7}

Planteamientos metodológicos para enseñar ciencias en Primaria. * Medias con diferentes letras en superíndice (a,b) difieren significativamente $(P<0,01)$

Ítem 7-CF2: Para enseñar ciencias, los planteamientos metodológicos que deberían utilizarse en Primaria son:

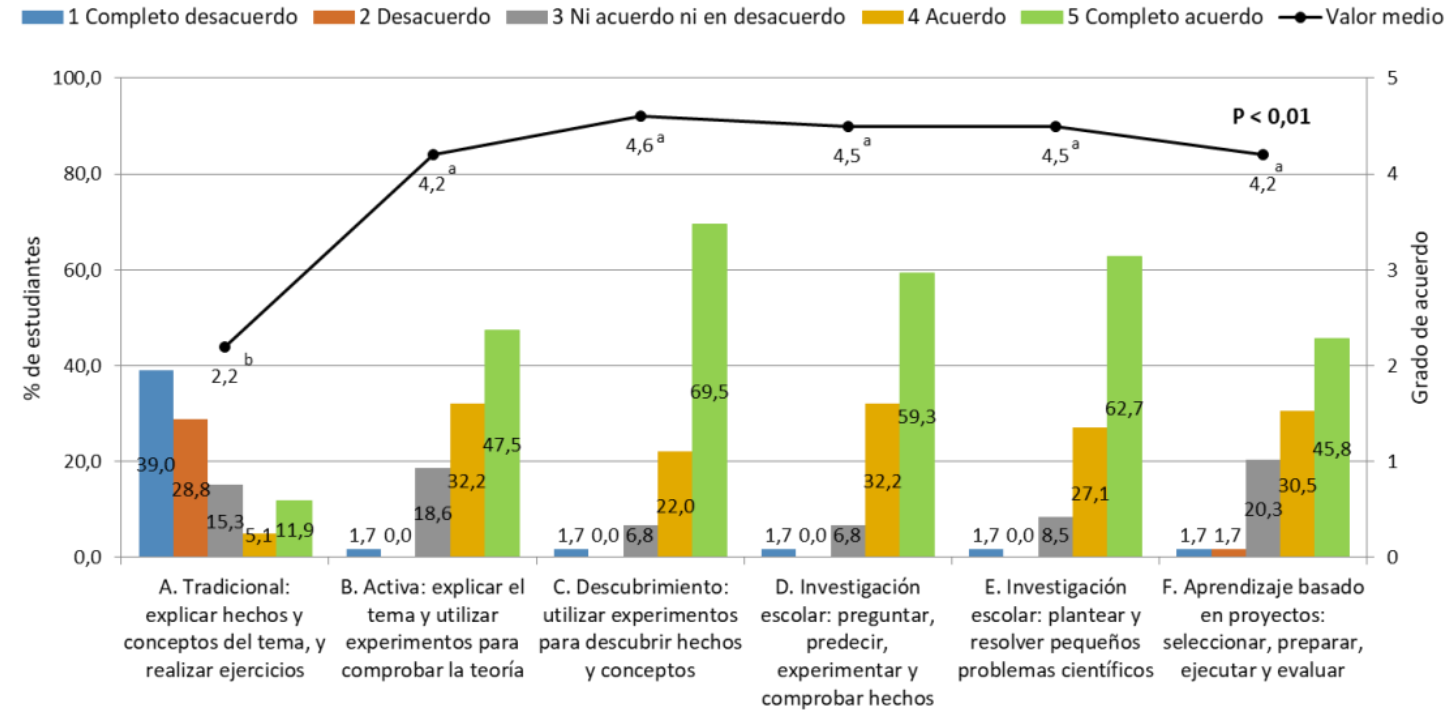


Esta diversidad en la elección de los planteamientos metodológicos más adecuados para enseñar ciencias, podría explicarse atendiendo a que se trata de estudiantes cuyas experiencias como alumnos han estado situadas en una metodología mayoritariamente tradicional, así como, que son alumnos que han finalizado el programa de formación en DCCEE, lo que podría haber influido en su elección, al estar situados los presupuestos del programa para dirigir el desarrollo de los contenidos en un enfoque constructivista mediante la exploración del alumno. En este sentido, trabajos anteriores señalan situaciones similares de desacuerdo con el enfoque tradicional «puro» y se afianza el acuerdo con el enfoque alternativo que se promueve desde el programa de formación (Hamed et al., 2016; Rivero et al., 2017).

\section{3. ¿Qué hacen los alumnos cuando enseñan las ciencias en primaria?}

\section{Qué actuaciones docentes realizan}

De los 59 participantes en el cuestionario CF2, 47 (79,7 \%) declaran haber enseñado ciencias en sus PE, encontrando que el 74,6 \% del grupo lo hace con su maestro tutor (bien en español o inglés) y el resto recurre al maestro especialista en inglés o a otro maestro del centro (tabla 6). Destacar que 12 estudiantes (20,3 \% del grupo) no realizan ninguna actuación docente en el área de ciencias, aludiendo a causas ajenas a sus decisiones, "mi tutor no enseñaba ciencias" o "las ciencias de la naturaleza se impartían en inglés", lo que constituyen causas externas a la actividad formativa. El hecho de no realizar actuaciones docentes por un número considerable de alumnos es algo que se repite en el tiempo, como manifiestan los estudiantes que responden al cuestionario CF1 el curso anterior (2016/17), donde 23 estudiantes $(31,9 \%)$ no realizan actuaciones docentes en ciencias. Cabe pensar, que el bilingüismo en la enseñanza de las ciencias agrava esta situación, pues limita el número de maestros tutores que enseñen ciencias o aumenta la posibilidad de que las ciencias se den inglés.

\section{Tabla 6}

Actuaciones docentes en ciencias (Ítem 8-CF2: En el colegio donde has realizado las prácticas escolares, ¿̇has enseñado Ciencias de la Naturaleza?; Item 1-CF1: ¿Has podido planificar y desarrollar alguna actuación docente en Ciencias de la Naturaleza?). Valores expresados como frecuencia (\%)

\begin{tabular}{lcccc}
\hline & \multicolumn{2}{c}{ Ítem 8-CF2 } & \multicolumn{2}{c}{ Ítem 1-CF1 } \\
Respuestas & Frecuencia & \% & Frecuencia & \% \\
\hline Si & 40 & 67,8 & 37 & 51,4 \\
$\quad \begin{array}{l}\text { Con mi tutor/a, en español } \\
\text { Con mi tutor/a en inglés }\end{array}$ & 4 & 6,8 & 2 & 2,8 \\
$\quad \begin{array}{l}\text { Con el maestro/a especialista, en } \\
\text { inglés }\end{array}$ & 2 & 3,4 & 7 & 9,7 \\
$\begin{array}{l}\text { Con otro maestro, en español, en un } \\
\text { curso diferente al asignado }\end{array}$ & 1 & 1,7 & 3 & 4,2 \\
\hline$\quad$ Total & 47 & 79,7 & 49 & 68,1 \\
\hline $\begin{array}{l}\text { No } \\
\quad \text { Mi maestro tutor no enseñaba }\end{array}$ & 8 & 13,5 & - & - \\
$\begin{array}{l}\text { Ciencias de la Naturaleza } \\
\text { Las Ciencias de la Naturaleza se }\end{array}$ & 2 & 3,4 & - & - \\
$\quad \begin{array}{l}\text { impartían en inglés } \\
\text { No he querido, he preferido enseñar } \\
\text { otras asignaturas }\end{array}$ & 2 & 3,4 & - & - \\
\hline$\quad$ Total & 12 & 20,3 & 23 & 31,9 \\
\hline
\end{tabular}

Con respecto al tipo de actuación docente que realizan (tabla 7), los alumnos se sitúan mayoritariamente en aquellas que son requeridas por el plan de PE, actividades puntuales y unidad de programación, seguido de otras actuaciones que requieren menor complejidad y que son tareas habituales de apoyo al maestro tutor (TAT) (corregir ejercicios, explicaciones puntuales,...), siendo en estas últimas donde participan los estudiantes que manifestaban no haber realizado actuaciones en ciencias. Un 40,7 \% (24 estudiantes) y un 18,6\% (11 estudiantes), diseña y pone en práctica una actividad puntual o una unidad de programación y reflexiona sobre su actuación docente. Por tanto, cerca del $60 \%$ de los alumnos optan por actuaciones comprometidas con la enseñanza de las ciencias. 
Tabla 7

Tipo de actuaciones docentes en ciencias (Ítem 9-CF2: Las actuaciones docentes que be realizado en Ciencias de la Naturaleza han sido). Valores expresados como frecuencia (\%)

\begin{tabular}{llcc}
\hline $\begin{array}{l}\text { Respuestas } \\
\text { ítem 8-CF2 }\end{array}$ & Respuestas ítem 9-CF2 & Frecuencia & \% \\
\hline Si & Actividad puntual del plan de PE & 19 & 32,2 \\
& Actividad puntual + TAT & 4 & 6,8 \\
& Actividad puntual plan PE + Unidad programación plan & 1 & 1,7 \\
& PE + TAT + varias actividades & 9 & 15,2 \\
& Unidad de programación del plan de PE & 1 & 1,7 \\
& Unidad programación + varias actividades puntuales & 9 & 15,2 \\
& TAT & 1 & 1,7 \\
& Varias actividades puntuales en diferentes lecciones & 3 & 5,1 \\
\hline TAT + Varias actividades puntuales & 47 & 79,7 \\
\hline \multirow{2}{*}{ No } & Total & 10 & 16,9 \\
& TAT & 2 & 3,4 \\
\hline & Varias actividades puntuales en diferentes lecciones & 12 & 20,3 \\
\hline
\end{tabular}

Atendiendo a las declaraciones que realizan los estudiantes que responden al cuestionario CF1, la utilidad del programa de formación en sus actuaciones, están vinculadas en mayor medida con las actuaciones más complejas y las requeridas por el plan de PE: unidad de programación $(34,7 \%)$ y actividades puntuales $(30,6 \%)$, seguida de las tareas docentes habituales de apoyo al maestro/a tutor/a $(16,7 \%)$ (tabla 8$)$.

Estos valores son más alentadores que los encontrados por Cortés et al. (2012) donde solo un 17 \% de los alumnos declaraban su utilidad para la preparación de actividades.

\section{Tabla 8}

Utilidad de la formación en DCCEE en el tipo de actuación docente de PE (Ítem 3-CF1: ¿Has podido aplicar la formación recibida en las asignaturas del Departamento de DCCEE durante tu actuación docente?)

\begin{tabular}{lcc}
\hline Respuestas & Frecuencia & $\mathbf{\%}$ \\
\hline Si, en una de las actividades puntuales & 22 & 30,6 \\
Si, en la unidad de programación & 25 & 34,7 \\
Si, durante las TAT & 12 & 16,7 \\
No he realizado actividades ni unidad de programación en CC & 21 & 29,2 \\
Otros & 1 & 1,4 \\
$\quad$ En el diseño de un examen sobre seres vivos & 1 & 1,4 \\
$\quad$ He usado detalles, el contenido no lo había trabajado en ninguna de las & & 1,4 \\
$\quad$ asignaturas de DCCEE & 1 & 1,4 \\
$\quad$ No he podido aplicar la formación & 1 & \\
$\quad$ No, durante mi periodo no hemos visto similar en la asignatura & & \\
\hline
\end{tabular}

La baja utilidad manifestada en las tareas docentes habituales, quizás sea debida a que no identifican que tras las rutinas profesionales de aula existen modelos educativos y didácticos, coherentes con marcos teóricos determinados sobre los procesos de enseñanza y aprendizaje de las ciencias. En cualquier caso, la reflexión que se les pide a los alumnos no contribuye a poner en valor el conocimiento académico recibido y establecer relaciones entre la dimensión teórica, práctica y profesional del conocimiento didáctico.

Con respecto a los conocimientos académicos relacionados con el análisis y diseño de actividades, destacados antes por su alta valoración, resultan útiles para los alumnos cuando tienen que seleccionar y planificar actividades de enseñanza o elaborar los materiales escritos para los alumnos, lo que responde al sentido dado en el programa de formación (figura 8). En menor medida, otros alumnos señalan que no les han sido útiles, incidiendo en causas externas al programa de formación. 


\section{Figura 8}

Utilidad de los conocimientos académicos sobre análisis y diseño de actividades de enseñanza en la PE

Ítem 4-CF1. ¿Los conocimientos recibidos en las asignaturas del departamento sobre análisis y diseño de actividades de enseñanza para el estudio de las Ciencias te han sido de utilidad?

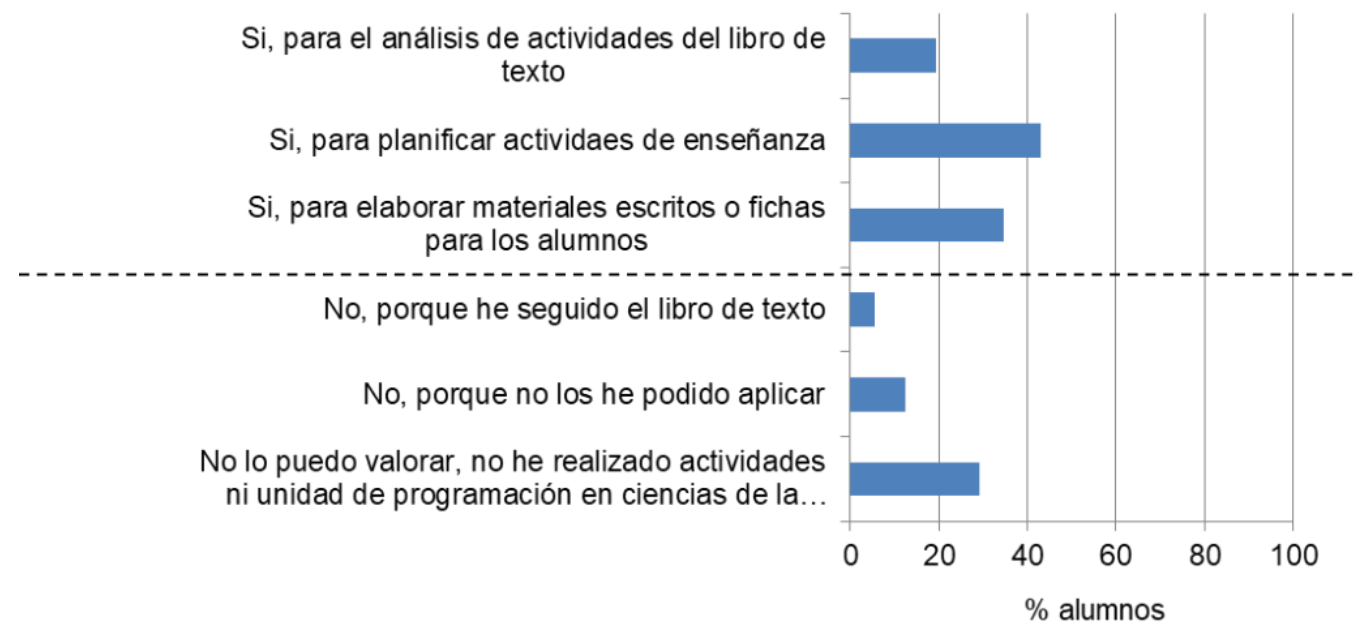

\section{Qué contenidos de ciencias enseñan}

En sus actuaciones docentes, los alumnos enseñan contenidos de todos los bloques curriculares (item 2-CF1: Si has planificado y desarrollado alguna actividad o unidad didáctica en Ciencias de la Naturaleza, ¿en qué bloque de contenidos ha sido?): los seres vivos (26.4\%), el ser humano y la salud $(23.6 \%)$, la materia y energía (22.2 \%), iniciación a la actividad científica (13.9\%) y la tecnología, los objetos y las máquinas $(4.2 \%)$. Dado que, según el plan de prácticas de $\mathrm{PE}$, los alumnos deben respetar las programaciones de los centros, los resultados responden a esta circunstancia. Destacar la escasa presencia de los contenidos del bloque 1 - iniciación a la actividad científica, pues no corresponde con el carácter transversal que se les ha asignado en el programa de formación cuando en los seminarios analizan y diseñan propuestas de enseñanza sobre cualquier tópico de ciencias; tampoco corresponde con los resultados anteriormente destacados sobre sus concepciones acerca de qué enseñar de ciencias en primaria (figura 6), donde destacan contenidos relacionado con actitudes hacia el medio ambiente y procesos científicos. Por tanto, cabe pensar que los logros del programa de formación no sean suficientes para que los alumnos que han diseñado actividades puntuales o unidades de programación hayan tenido en cuenta estos presupuestos. Por último, un $26.4 \%$ no contestó esta pregunta.

\section{Cómo enseñan las ciencias}

La mayoría de los alumnos identifican su forma de enseñar con una metodología transmisiva, tradicional o activa, donde primero se dan explicaciones y después se realizan ejercicios o experimentos para comprobar la teoría (Figura 9). Esta situación es similar a la encontrada en trabajos anteriores con maestros en formación (Cañal et al., 2013; De Pro y Nortes, 2016). Sólo unos pocos consideran que han realizado una investigación escolar o un proyecto. 


\section{Figura 9}

Metodología utilizada para enseñar ciencias en las PE. Valores expresados como frecuencia (\%)

Ítem 10-CF2. Las actuaciones docentes que he realizado corresponden con la siguiente forma de enseñar:

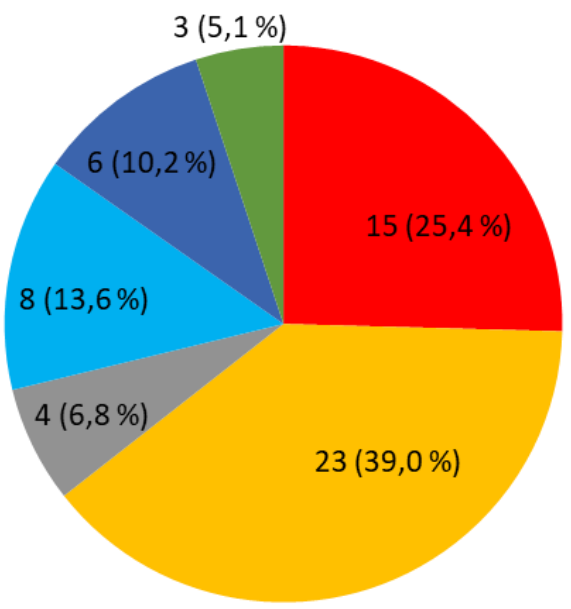

A. Tradicional: explicar hechos y conceptos del tema, y realizar de ejercicios

B. Activa: explicar el tema y utilizar experimentos para comprobar la teoría

c. Descubrimiento: utilizar experimentos para descubrir hechos y conceptos

D. Investigación escolar: preguntar, predecir, experimentar y comprobar hechos

- E. Investigación escolar: plantear y resolver pequeños problemas científicos

- F. Aprendizaje basado en proyectos: seleccionar, preparar, ejecutar y evaluar

Estos resultados contrastan fuertemente con sus opiniones sobre cómo enseñar ciencias en primaria (figuras 7), tanto por el rechazo que señalan a la enseñanza tradicional, como por elegir enfoques de enseñanza práctica (descubrimiento e investigación escolar, en mayor medida) como la forma de enseñanza deseable. Esta falta de correspondencia entre lo que piensan y lo que hacen ha sido señalada en trabajos anteriores (Fernández et al., 2009; Mellado, 1998b; Weissmann, 1993). Atendiendo a nuestros resultados, creemos que puede deberse a que los maestros en formación perciben un predominio de enseñanza tradicional en las aulas de primaria, lo que se justifica por los resultados obtenidos sobre la adecuación de la metodología utilizada (figura 10), donde el 60,0 \% (9 alumnos) y 65,2 \% (15 alumnos) de los alumnos que indicaban haber enseñado de forma tradicional o activa, respectivamente, responden que es adecuada porque «era la utilizada por su tutor/a» o «es a la que están acostumbrados los alumnos»; pero también, a bajos niveles de autoeficacia percibida por los alumnos, lo que favorece que cuando se ven desbordados activen las rutinas tradicionales para no perder el control de la clase (Porlán, 2018). Este hecho se pone de manifiesto, cuando los alumnos reconocen inadecuada la metodología utilizada, señalando inseguridad para cambiar las rutinas de aula, la falta de tiempo y/o de material; así como, cuando responden «es la que están acostumbrados los alumnos», la cual permite inferir escasa necesidad de ensayar nuevos métodos para responder más adecuadamente a las necesidades de sus alumnos.

\section{Figura 10}

Adecuación de la metodología utilizada en las PE para enseñar ciencias. Valores expresados como frecuencia (\%) 
Ítem 11-CF2. La metodología que he utilizado para enseñar considero que ha sido:

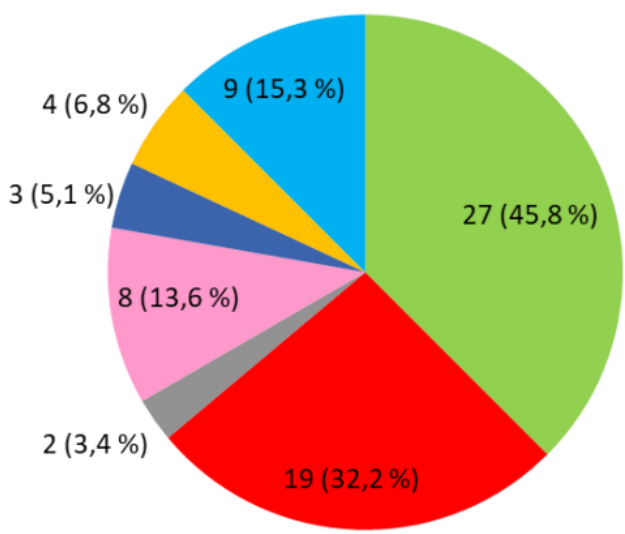

A. Adecuada, porque era la que utilizaba mi tutor/a

- B. Adecuada, porque es a la que están

acostumbrados los alumnos

C. Adecuada, porque es la que propone el libro de

texto

D. Inadecuada, porque no es la forma ideal de

enseñar ciencias

E. Inadecuada, porque no he podido realizar cambios

por falta de tiempo, material, ...

F. Inadecuada, porque me habría gustado enseñar de

otra forma pero me sentía inseguro

G. Por otra razón

Cabe pensar que los alumnos tienen un grado de autonomía profesional bajo durante las PE, pero también que no perciben la necesidad de introducir cambios metodológicos, aunque en el plan de prácticas se les plantea la conveniencia de realizar innovaciones didácticas. En el contexto de las PE, el modelo de enseñanza percibido por los alumnos en las aulas y su bajo nivel de autoeficacia mostrado, parecen tener un peso mayor que el conocimiento académico vinculado al programa de formación (DCCEE) en su conducta docente, poniendo de manifiesto las débiles relaciones entre los conocimientos académicos y profesionales, como ya apuntaba Porlán et al. (2010, 2011), así como, la necesidad de mejorar la autoeficacia de los futuros docentes para favorecer prácticas docentes innovadoras durante el proceso de enseñanza en las PE y que perduren en el tiempo como señalan algunas investigaciones (Brand y Wilkins, 2007; Palmer, 2007).

\section{Conclusiones e implicaciones}

El estudio sobre cuáles son las ideas de los futuros maestros sobre el programa de formación, sus concepciones sobre enseñar ciencias y su repercusión sobre lo que hacen en las prácticas escolares ofrece unos resultados que, aunque aparentemente contradictorios, evidencian las singularidades de ambos contextos formativos. Así los resultados muestran una valoración positiva o muy positiva del programa de formación en su conjunto tanto por su contenido de enseñanza como por las actividades formativas realizadas. Los futuros maestros se identifican con concepciones sobre la enseñanza de las ciencias en primaria coherentes con los presupuestos del programa de formación, pero con planteamientos metodológicos, que aun adoptando ideas de un enfoque constructivista e investigativo, todavía mantienen concepciones del modelo transmisivo.

En cuanto a las actuaciones de los alumnos durante las prácticas escolares, estas están alejadas de los presupuestos anteriores. Por un lado, el contexto escolar (maestro tutor sin carga lectiva en ciencias, bilingüismo en la asignatura de ciencias, entre otras) reduce la posibilidad de los alumnos de tener oportunidad de llevar a cabo actuaciones relacionadas con la enseñanza de las ciencias. Por otro, el modelo de enseñanza tradicional percibido por los maestros en formación inicial en las aulas, así como, su bajo nivel de autoeficacia para realizar prácticas docentes innovadoras, no contribuyen a poner en valor el conocimiento académico vinculado al programa de formación (DCCEE) y establecer relaciones entre los conocimientos académicos teóricos y prácticos y la práctica profesional.

Por tanto, dentro de los planes de estudio actuales, las acciones formativas no son suficientes para promover cambios deseables en las prácticas docentes de los alumnos durante su formación inicial cuando enseñan ciencias. Las posibles mejoras pasan por prestar mayor atención a las prácticas escolares tanto para superar los obstáculos externos como para buscar las condiciones que posibiliten una mayor transferencia de los conocimientos académicos teóricos y prácticos a las aulas.

Consideramos que es necesario vincular la teoría y la práctica atendiendo a aspectos que requieren: 1) un compromiso colectivo del centro escolar de prácticas, que permita por un lado superar los obstáculos externos y los hagan adecuados para recibir estudiantes de prácticas, así como, proporcionar maestros tutores comprometidos con una práctica innovadora y coherente con los presupuestos del programa formativo que se imparte en el grado de maestro; 2) la colaboración compartida entre los tutores 
universitarios, maestros tutores del centro escolar y los futuros maestros, de manera que se conformen equipos de trabajo que tengan como objetivo prioritario desarrollar todos los elementos que ayuden a los futuros maestros a construir su identidad profesional como indican Deulofeu et al. (2010), así como, formar maestros en un ambiente colaborativo, reflexivo y crítico, que posibilite la construcción activa de sus conocimientos sobre la práctica docente (Alsina y Batllori, 2015; Casas et al., 2004), y, por otro lado, mejore las creencias de autoeficacia de los estudiantes mediante el fortalecimiento del sentido de eficacia colectiva (Goddard et al., 2004).

\section{Referencias}

Alsina, Á. y Batllori, R. (2015). Hacia una formación del profesorado basada en la integración entre la práctica y la teoría: una experiencia en el Prácticum desde el modelo realista. Investigación en la Escuela, 85, 5-18. DOI: 10.12795/IE.2015.i85.01

Blonder, R., Benny, N. y Jones, M.G. (2014). Teaching self-efficacy of science teachers. En R.H. Evans, J. Luft, C.Czerniak, \& C. Pea (Eds.), The Role of Science Teachers' Beliefs in International Classrooms (pp. 3-15). SensePublishers.

Bonil, J. y Márquez, C. (2011). ¿Qué experiencias manifiestan los futuros maestros sobre las clases de ciencias? Implicaciones para su formación. Revista de Educación, 354, 447-472. DOI: 10.4438/1988592X-RE-2011-354-007.

Bozu, Z. y Aránega, S. (2017). La formación inicial de maestros y maestras a debate: ¿Qué nos dicen sus protagonistas?. Profesorado, Revista de Curriculum y Formación del Profesorado, 21(1), 143-163.

Brand, B.R. y Wilkins, J.L.M. (2007). Using self-efficacy as aconstruct for evaluating science and mathematics methods courses. Journal of Science Teacher Education, 18, 297-317. DOI: 10.1007/s10972007-9038-7.

Cañal, P. (2000). El conocimiento profesional sobre las ciencias y la alfabetización científica en primaria. Alambique, 24, 46-56.

Cañal, P., Criado, A.M., Ruiz, N.J. y Herzel, C. (2008). Obstáculos y dificultades de los maestros en formación en el diseño de unidades didácticas de enfoque investigador: el inventario general de obstáculos. En M.R. Jiménez Liso (Ed.), Ciencias para el mundo contemporáneo y formación del profesorado en Didáctica de las Ciencias Experimentales (pp. 344-353). Almería: Universidad de Almería.

Cañal, P. (2012). ¿Cómo evaluar la competencia científica? Investigación en la escuela, 78, 5-17. DOI: 10.12795/IE.2012.i78.01.

Cañal, P., Criado, A.M., García-Carmona, A. y Muñoz, G. (2013). La enseñanza relativa al medio en las aulas españolas de Educación Infantil y Primaria: concepciones didácticas y práctica docente. Investigación en la Escuela, 81, 21-42. DOI: 10.12795/IE.2013.181.02.

Casas, M., Creus, T., Valls, C., Bosch, M. D., Bonil, J., Camps, A., Font, R., López, L., Prat, M., Ribas, T. y Sanmartí, N. (2004). Un Practicum per a la formació de mestres innovadors, reflexius i crítics. Jornades de Camppus d'innovació Docent, 1-4.

Cortés, A., De la Gándara, M., Calvo, J., Martínez, M. y Gil, M. (2012). Expectativas, necesidades y oportunidades de los maestros en formación ante la enseñanza de las ciencias en la educación primaria. Enseñanza de las ciencias, 30(3), 155-176.

De Pro, A. y Nortes, R.M. (2016). ¿Qué pensaban los estudiantes de la diplomatura de maestro de educación primaria sobre las clases de ciencias de sus prácticas de enseñanza? Enseñanza de las ciencias, 34(1), 7-32. DOI: $10.5565 / \mathrm{rev} /$ ensciencias. 1762.

Deulofeu, J., Conxita, M. y Sanmartí, N. (2010). Formar profesores de secundaria. La experiencia de la Universitat Autónoma de Barcelona. Cuadernos de Pedagogía, 404, 80-84.

Escobar, T. y Vílchez, J. E. (2008). Percepción de los estudiantes de magisterio durante el prácticum sobre las clases reales de ciencias de educación primaria. En M. R. Jiménez (ed.). Ciencias para el mundo contemporáneo y formación del profesorado en Didáctica de las Ciencias Experimentales (pp. 583-592). Universidad de Almería.

Fernández, M.T., Tuset, A.M., Pérez, R.E. y A.C. Leyva (2009). Concepciones de los maestros sobre la enseñanza y el aprendizaje y sus prácticas educativas en clases de ciencias naturales. Enseñanza de las Ciencias, 27(2), 287-298.

García-Carmona, A., Cruz-Guzmán, M. y Criado, A. (2014). ¿Qué hacías para aprobar los exámenes de Ciencias, qué aprendiste y qué cambiarías?. Preguntamos a futuros docentes de Educación Primaria. Investigación en la Escuela, 84, 31-46. DOI: 10.12795/IE.2014.i84.03. 
García-Carmona, A. y Cruz-Guzmán, M. (2016). ¿Con qué vivencias, potencialidades y predisposiciones inician los futuros docentes de Educación Primaria su formación en la enseñanza de la ciencia?. Revista Eureka sobre Enseñanza y Divulgación de las Ciencias, 13(2), 440-458. DOI: 10498/18299.

Gil Flores, J. (2016). Variables asociadas a la autoeficacia percibida por el profesorado de ciencias en Educación Secundaria. Revista de Educación, 373, 85-108. DOI: 10.4438/1988-592X-RE-2016-373-322

Goddard, R. D., Hoy, W. K. y Woolfolk Hoy, A. (2004). Collective efficacy beliefs: Theoretical developments, empirical evidence, and future directions. Educational Researcher, 33(3), 3-13. DOI: 10.3102/0013189X033003003.

Gunning, A.M. y Mensah, F.M. (2011). Preservice elementary teachers' development of self-efficacy and confidence to teach science: A case study. Journal of Science Teacher Education, 22, 171-185. DOI: 10.1007/s10972-010-9198-8.

Hamed, S., Rivero, A. y Martín del Pozo, R. (2016). El cambio en las concepciones de los futuros maestros sobre la metodología de enseñanza en un programa formativo. Revista Eureka sobre Enseñanza y Divulgación de las Ciencias, 13(2), 476-492.

King, K., Shumow, L. y Lietz, S. (2001). Science education in an urban elementary school: Case studies of teacher beliefs and classroom practices. Science Education, 85, 89-110. DOI:10.1002/1098237X(200103) 85:2<89::AID-SCE10>3.0.CO;2-H

Martínez-Losada, C., García-Barros, S. y Rivadulla, J.C. (2017). Percepción de los maestros en formación sobre los objetivos de enseñanza y sus propias capacidades profesionales. X Congreso internacional sobre investigación en didáctica de las ciencias, Enseñanza de las ciencias, N. ${ }^{\circ}$ Extraordinario, 123-128. Recuperado de https://www.raco.cat/index.php/Ensenanza/article/view/333975.

Mellado, V. (1998a). Preservice teachers' classroom practice and their conceptions of the nature of science. En B. J. Fraser y K. Tobin (eds.), International Handbook of Science Education (pp. 1093-1105). Kluwer Academic Publishers.

Mellado, V. (1998b). The classroom practice of preservice teachers and their conceptions of teaching and learning science. Science Teacher Education, 82, 197-214. DOI: 10.1002/(SICI) 1098237X(199804)82:2<197::AID-SCE5>3.0.CO;2-9

Mellado, V. (1999). La investigación sobre la formación del profesorado en Ciencias Experimentales. En C. Martínez y S. García (eds.), La Didáctica de las Ciencias. Tendencias actuales. XVIII Encuentros de didáctica de las Ciencias Experimentales (pp. 45-76). Universidad da Coruña.

Mellado, V. y González, T. (2000). La formación inicial del profesorado de ciencias. En F.J. Perales y P. Cañal (Ed.). Didáctica de las Ciencias Experimentales (pp. 535-555). Marfil.

Mellado, V. (2001). ¿Por qué a los profesores de ciencias nos cuesta tanto cambiar nuestras concepciones y modelos didácticos? Revista Interuniversitaria de Formación del Profesorado, 40, 17-30.

Palmer, D. (2007). Durability of changes in self-efficacy of preservice primary teachers. International Journal of Science Education, 28, 655-671. DOI: 10.1080/09500690500404599.

Parker, J. y Spink, E. (1997) Becoming Science Teachers: an evaluation of the initial stages of primary teacher training. Assessment $y$ Evaluation in Higher Education, 22(1), 17-31, DOI: 10.1080/0260293970220102.

Pedrinaci, E.; Caamaño, A.; Cañal, P. y Pro, A. (2012). La evaluación de la competencia científica requiere nuevas formas de evaluar los aprendizajes. En E. Pedrinaci (Coord.), 11 ideas clave: El desarrollo de la competencia cientifica (pp. 241-267). Graó.

Petersen, J. E. y Treagust, D. F. (2014). School and University Partnerships: The Role of Teacher Education Institutions and Primary Schools in the Development of Preservice Teachers' Science Teaching Efficacy. Australian Journal of Teacher Education, 39(9), 153-167. DOI: $10.14221 /$ ajte.2014v39n9.2.

Porlán, R., Martín del Pozo, R., Rivero, A., Harres, J., Ázcárate, P. y Pizzato, M. (2010). El cambio del Profesorado de Ciencias I: Marco Teórico y Formativo. Enseñanza de la Ciencias, 28(1), 31-46.

Porlán, R., Martín del Pozo, R., Rivero, A., Harres, J., Azcárate, P. y Pizzato, M. (2011). El cambio del profesorado de ciencias II: Resultados y conclusiones sobre la progresión de las concepciones didácticas. Enseñanza de las Ciencias, 29(3), 413-426.

Porlán, R. (2018). Didáctica de las ciencias con conciencia. Enseñanza de las ciencias, 36(3), 5-22. https://doi.org/10.5565/rev/ensciencias.2795.

Pujol, R. M. (2008). Pensar en la escuela primaria para pensar en la formación de su profesorado, desde la DCE, en el marco del nuevo grado. En: M.R. Jiménez Liso (Ed.), Ciencias para el mundo contemporáneo y formación del profesorado en Didáctica de las Ciencias Experimentales (pp. 354-361). Universidad de Almería. 
Real Decreto 126/2014, de 28 de febrero, por el que se establece el currículo básico de la Educación Primaria. Boletín Oficial del Estado, 52, de 1 de marzo 2014. Recuperado de https://www.boe.es/buscar/pdf/2014/BOE-A-2014-2222-consolidado.pdf.

Richardson, G.M. y Lian, L.L. (2008). The use of inquiry in the development of preservice teacher efficacy in Mathematics and Science. Journal of Elementary Science Education, 20(1), 1-16. DOI:10.1007/BF03174699.

Rivero, A., Solís, E., Porlán, R., Azcárate, P. y Martín del Pozo, R. (2017). Cambio del conocimiento sobre la enseñanza de las ciencias de futuros maestros. Enseñanza de las Ciencias, 35(1), 29-52. https://doi.org/10.5565/rev/ensciencias.2068.

Ruiz-Bernardo, P., Sanchez-Tarazaga, L. y Mateu-Pérez, R. (2018). La innovación pedagógica de la mano de la investigacion-acción para mejorar la calidad de las prácticas externas de los Grados de Maestro/a en Educación Primaria y Educación Infantil. Revista Electrónica Interuniversitaria de Formación del Profesorado, 21(1), 33-49. DOI: 10.6018/reifop.21.1.277681.

Sánchez, G. y Valcarcel, M.V. (2000). Relación entre el conocimiento científico y el conocimiento didáctico del contenido: un problema en la formación inicial del profesor de secundaria. Alambique, 24, 76-78.

Smith, L. y Southerland, S. A. (2007). Reforming practice or modifying reforms? Elementary teachers' response to the tools of reform. Journal of Research in Science Teaching, 43(3), 396-423. DOI 10.1002/tea.20165.

Tuli F. y File, G. (2009). Practicum Experience In Teacher Education. Ethiopian Journal of Education and Sciences, 5(1), 107-116. DOI: 10.4314/ejesc.v5i1.56316.

Velthuis, C., Fisser, P. y Pieters, J. (2013). Teacher training and pre-service primary teachers' self-efficacy for science teaching. Journal of Science Teacher Education, 25, 445-464. DOI: 10.1007/s10972-013-9363y.

Vílchez, J. y Escobar, T. (2014). Uso de laboratorio, huerto escolar y visitas a centros de naturaleza en Primaria: Percepción de los futuros maestros durante sus prácticas docentes. Revista Electrónica de Enseñanza de las Ciencias, 13(2), 222-241.

Weissmann, H. (1993). Qué enseñan los maestros cuando enseñan ciencias naturales, y qué dicen querer enseñar. En H. Weissmann (Comp.), Didáctica de las Ciencias Naturales. Aportes y Reflexiones (pp. 37-65). Paidós. 